\title{
PID CONTROLLERS DESIGN APPLIED TO POSITIONING OF BALL ON THE STEWART PLATFORM
}

\author{
Andrzej KOSZEWNIK*, Kamil TROC ${ }^{*}$, Maciej SŁOWIK* \\ *Faculty of Mechanical Engineering, Bialystok University of Technology, ul. Wiejska 45C, 15-351 Białystok, Poland \\ a.koszewnik@pb.edu.pl, kamiltroc@o2.pl, m.slowik@pb.edu.pl
}

\begin{abstract}
The paper presents the design and practical implementation of PID controllers for a Stewart platform. The platform uses a resistance touch panel as a sensor and servo motors as actuators. The complete control system stabilizing the ball on the platform is realized with the Arduino microcontroller and the Matlab/Simulink software. Two processes required to acquire measurement signals from the touch panel in two perpendicular directions $\boldsymbol{X}$ and $\boldsymbol{Y}$, are discussed. The first process includes the calibration of the touch panel, and the second process - the filtering of measurement signals with the low pass Butterworth filter. The obtained signals are used to design the algorithm of the ball stabilization by decoupling the global system into two local subsystems. The algorithm is implemented in a soft real time system. The parameters of both PID controllers (PID $\boldsymbol{x}$ and PIDy) are tuned by the trial-error method and implemented in the microcontroller. Finally, the complete control system is tested at the laboratory stand.
\end{abstract}

Key words: PID Controller, Low Pass Butterworth Filter, Stewart-Gough Platform, Arduino Microcontroller, USB Port, Touch Panel Sensor

\section{INTRODUCTION}

Parallel link manipulators known as the "Stewart-Gough" platforms (see Fig.1) were investigated thoroughly in the last years. Since their potential advantages as: high rigidity, high accuracy, and high load-carrying capacity they seem to be better in comparison to the conventional serial link manipulators. That is why many researches often choose such structures as control plants. The first parallel mechanism was introduced by Gough and Whitehall in 1956 year (Gough and Whitehall, 2012) as a tyre-testing machine. Next, the design of the platform was improved by Stewart in 1965. Then, the modernized structure was used as an aircraft or vehicle simulation device. Parallel manipulators were used also as platforms for communication satellite dishes and telescopes positioning.

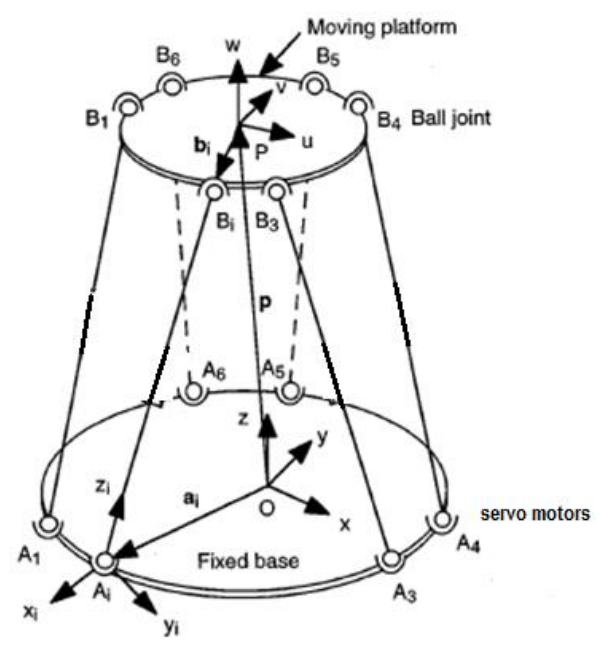

Fig. 1. Electrically actuated Stewart-Gough platform
The most popular type of actuators used in parallel link manipulators are pneumatic or hydraulic actuators. Both types of actuators have many advantages and their application in the system depends on the payload to weight ratio and on the dimensions of the system. Since pneumatic devices are less sensitive to temperature changes and contamination, a lot of researchers have tried controlling air cylinders using various methods.

One of the most popular control methods is the PID technique. Wang and Van Varseveld and Bone (Wang et al., 1999; Varseveld and Bone, 1997) used PID controllers with nonlinear compensation for servo-pneumatic actuators to control the position of the Stewart platform. Weng and Xu (2013) also used the PID controller with the LQG filter to control the position and to damp the vibration of the platform. Other researchers used non-traditional control methods. Urenizezius et al. applied the LQR technique. Paul et al implemented the sliding mode control (Urniezius and Geguzis, 2014; Paul et al., 1994; Shunmugham and Hayakawa, 1997). Wang et al. (2009) used the optimal control method based on the generalized natural frequencies of the system to stabilize the platform.

The Stewart platform discussed in the current article is a small size structure (the dimension of the fixed platform is $30 \mathrm{~cm}$, and of the moving platform - $11 \mathrm{~cm}$ ) with the low payload to weight ratio. The control system for a ball on the platform is realized by six electrical servo motors, the resistance touch panel and the PID controller. The choice of the PID control algorithm has been dictated mainly by practice problems of controlling the platform rather than by theoretical considerations of designing the control law. Other control methods would be difficult to implement because the mathematical model of the platform does not exist yet. Moreover, the PID control technique is a first control law investigated at this laboratory stand and it is realized together with a student. The design and experimental verification of other control methods will be the subject of future investigations. 
The paper presents consecutive steps of the design process and practical implementation of the chosen control algorithm for the platform and the Butterworth filters for the measurement signals.

\section{THE STEWART PLATFORM AS A PLANT OF THE CONTROL SYSTEM}

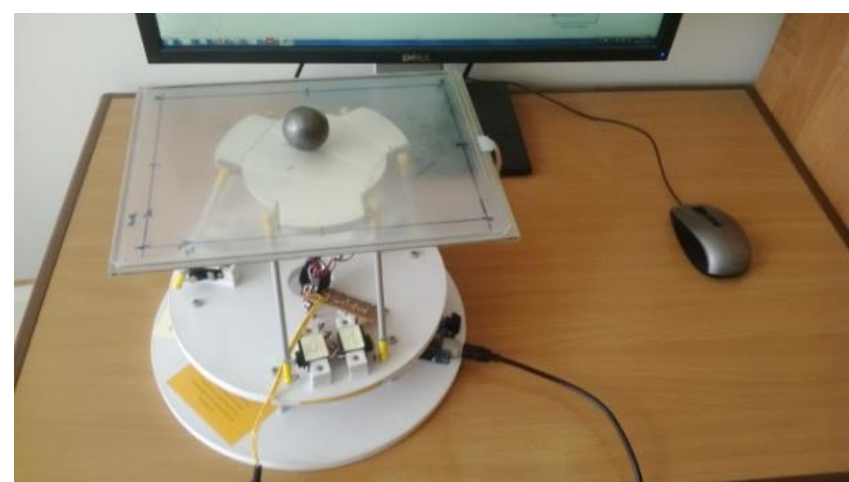

Fig. 2. The laboratory stand with the touch panel and the microcontroller
The 6-DOF Stewart platform shown in Fig. 2 consists of: a base frame, a moving platform, six legs, and components of the control system. As actuators the NewPower XL-9HM servomotors have been chosen and located on the frame base. As a sensor the RES121-4W touchpad has been chosen (http://www.buddyrc.com/new-power-xl-9hm.html). The process of stabilization of the ball on the upper platform is realized by the 8-bit Arduino Mega 2560 microcontroller operating at $16 \mathrm{MHz}$ frequency.

\section{THE CONTROL ALGORITHM}

The control algorithm of stabilization of the ball on the upper platform is realized by the established communication link between the Arduino microcontroller and the Matlab/Simulink software via the USB port. The standard transmission rate for this port is up to $480 \mathrm{Mb} / \mathrm{s}(115 \mathrm{~kb} / \mathrm{s}$ in the current case), so this rate is sufficient to control the system in the soft real time system regime (http://stackoverflow.com/questions/17308956/differencesbetween-hard-real-time-soft-real-time-and-firm-real-time). The diagrams of the control system and algorithm are shown in Figs. 3 and 4 .

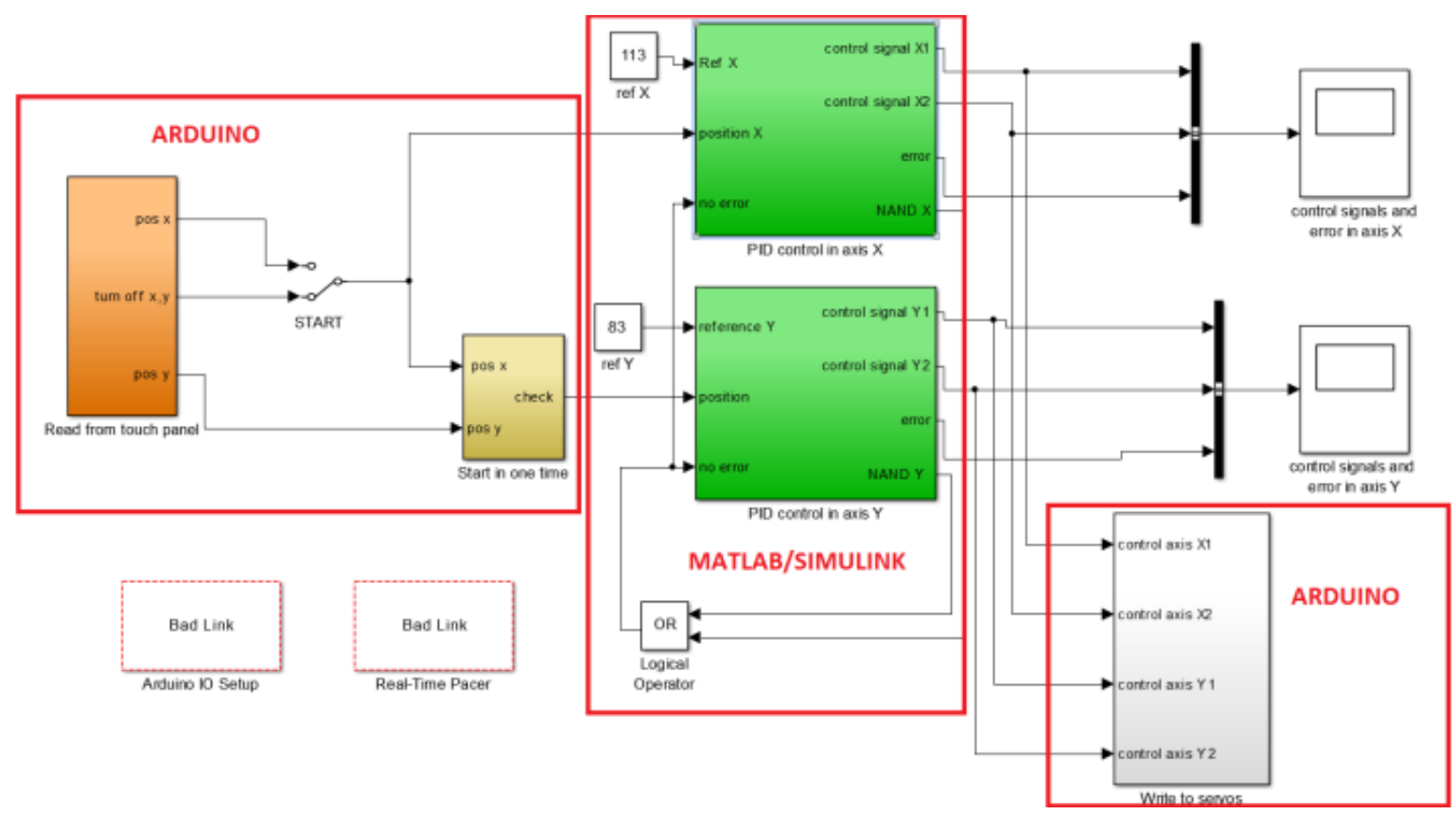

Fig. 3. The control scheme of the ball position

According to Fig. 4, the error signals in two perpendicular directions $X$ and $Y$ are obtained based on two measured signals (posx and posy) and two setpoint values $\left(r e f_{x}\right.$ and $\left.r e f_{y}\right)$. Both desired values: $r e f_{x}=113$ and $r e f_{y}=3$ correspond to a real middle point of the touch panel in two directions $X$ and $Y$, respectively. If the ball's position on the platform is greater than $2 \mathrm{~mm}$ in each direction then the control signals $u_{x}$ and $y$ are calculated in Matlab/Simulink and configuration of servo motors is changed (the signals are sent to Arduino). Otherwise the configuration of the servo motors remains the same.
The complete control system is based on the Matlab/Simulink software and the Arduino microcontroller hardware. As a result the block diagram consists four main subystems are prepared and shown in Fig.4:

- read from touchpanel - the signals from touchpanel is read by Arduino and filtered,

- simultaneous start - synchronous switch on both control feedback loop,

- write to servos - the values control signals from Matlab are recalculated and pass to servos,

- PID control - the control signals $u_{x}$ and $u_{y}$ are calculated with using Matlab software. 


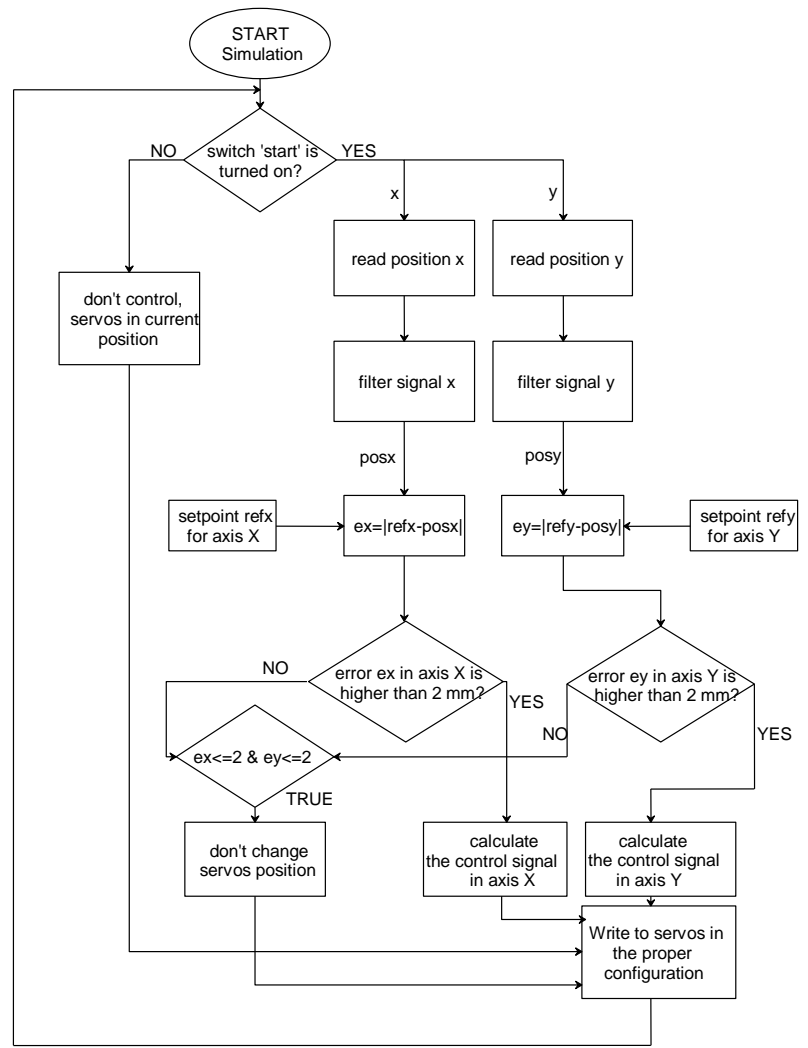

Fig. 4. Scheme algorithm of stabilization the ball on the platform $\left(r e f_{x}=113 \mathrm{~mm}, r e f_{y}=83 \mathrm{~mm}\right)$

\section{CALIBRATION PROCESS OF THE TOUCH PANEL}

The calibration process of the touch panel for different location of the ball on the platform is carried out in the paper. In this order the ball is located into two extreme position and one position into centre point on the sensor versus two perpendicular directions $X$ and $Y$, respectively. In results values of resistance of electrodes on the touch panel are measured and recorded in Fig. 5 and Fig. 6. Finally, obtained results are collected and transformed into displacement in Tab. 1.

Tab. 1. Results of calibration process of the touch panel

\begin{tabular}{|c|c|c|}
\hline Direction & Resistance $\Omega$ & Displacement $\mathrm{mm}$ \\
\hline$X$ & $207-875$ & $0-232$ \\
\hline$Y$ & $219-814$ & $0-166$ \\
\hline
\end{tabular}

The comparison of both figures (Fig. 5 and Fig. 6) shown correctly response action of the touch panel in both directions, but obtained values of resistance in $Y$ direction have firmly distribution. Thus, in order to design correctly control law these signals should pass by low pass 2-order Butterworth filter in frequency range up to $\omega_{\text {cutoff }}=35 \mathrm{rad} / \mathrm{s}$, which transfer function as follows (http://www.ece.uah.edu/corses/ee426/Butterworth.pdf):

$H(s)=\frac{0.1225}{s^{2}+0.495 s+0.1225}$

Taking into account Fig. 7 and Fig. 8 we can see that the signals from the touch panel in perpendicular directions are filtered, however application of this filter in the control system cause appear time delay on level $40 \mathrm{~ms}$. In results we obtained possibility of design the control system only in soft real-time regime system.

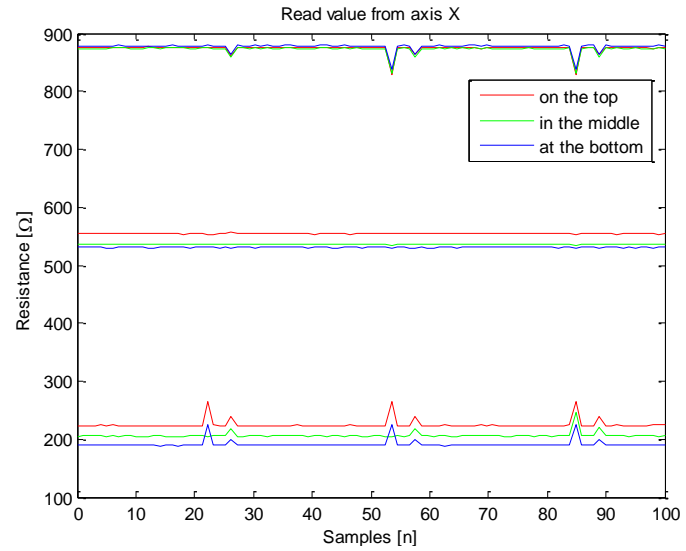

Fig. 5. The calibration of the touch panel in $X$ axis

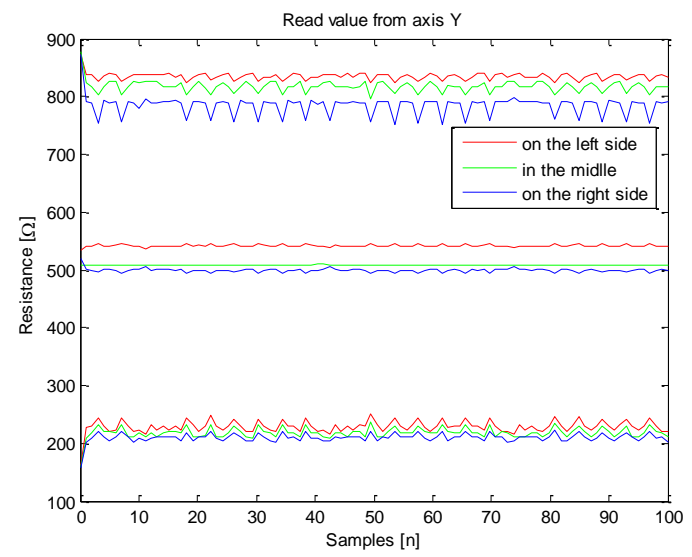

Fig. 6. The calibration of the touch panel in $Y$ axis
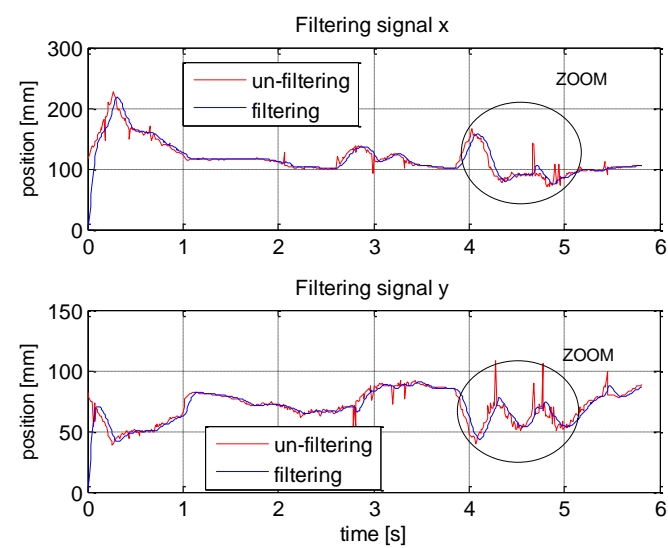

Fig. 7. The comparison of non-filtering and filtering signals
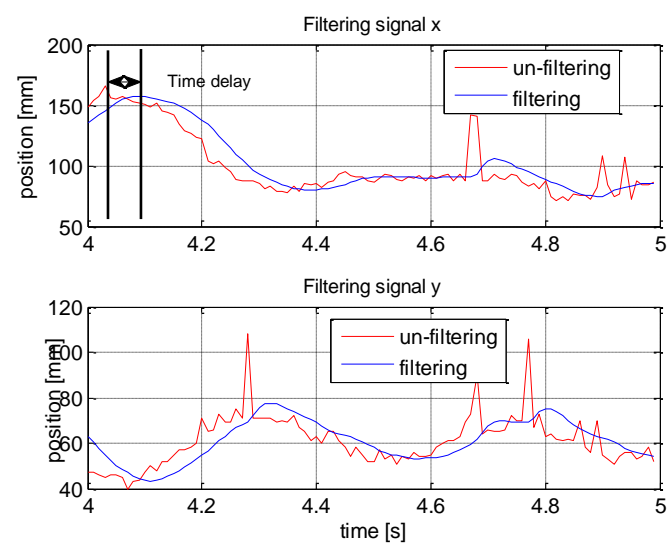

Fig. 8. The comparison of non-filtering and filtering signals in zoom scale 


\section{CONTROL STRATEGY}

The stage of design of stabilization the ball on the platform require to consider location of all six servo motors on the base platform. As first, the opposite site servo motors are coupled versus their location on the fixed base. Next, taking into account one couple of servo motors, the control signals $U+$ and $U-$ are generated by controllers for each of them. The control signals expressed by Eq. 2 consists value of 90 that represents the initial value of the control signal. Based on this assumption the control signals can be written as follows:

$$
\begin{aligned}
& U 1^{+}(t)=90+u_{P I D x}(t) \\
& U 1^{-}(t)=90-u_{P I D x}(t)
\end{aligned}
$$

where: $u_{P I D x}(t)=k_{p} e(t)+k_{i} \int e(t) d t+k_{d} \frac{d e(t)}{d t}$.

Practical realization of control subsystem to stabilize position of the ball in the $X$ axis is shown in Fig. 9. The subsystem accord- ing to Fig. 3 consists three inputs: $r e f_{x}=113$ (desired value), measurement value of position of the ball and signal without error. The last signal is responsible for simultaneously switching the control system between two directions for two cases. Once the ball is in the center point of the touch panel with extreme values less from $+/-2 \mathrm{~mm}$, but in the second case button START is switch off. If the ball is in the center point of the touch panel then values of the error signals are checked. If $-2<e x<2$ for $X$ direction then the output signal from logic gate $N A N D_{x}$ equal 0 and output value with logic gate $O R$ also is 0 (in case that $N A N D_{y}$ also equal 0 ). In results the error signal is 0 and all servo motors have still actual values. In second case the error signal is omitted if mentioned button has been switched off. In such case variable posx equals -20 and in the result one of inputs of multiply block is equal 0 . Finally, the control signals $X 1$ and $X 2$ for $X$ axis are generated by PIDx. Obtained signals in range of $30-150$ corresponds to minimal and maximal pitch angle of rudder bar of the servo motors.

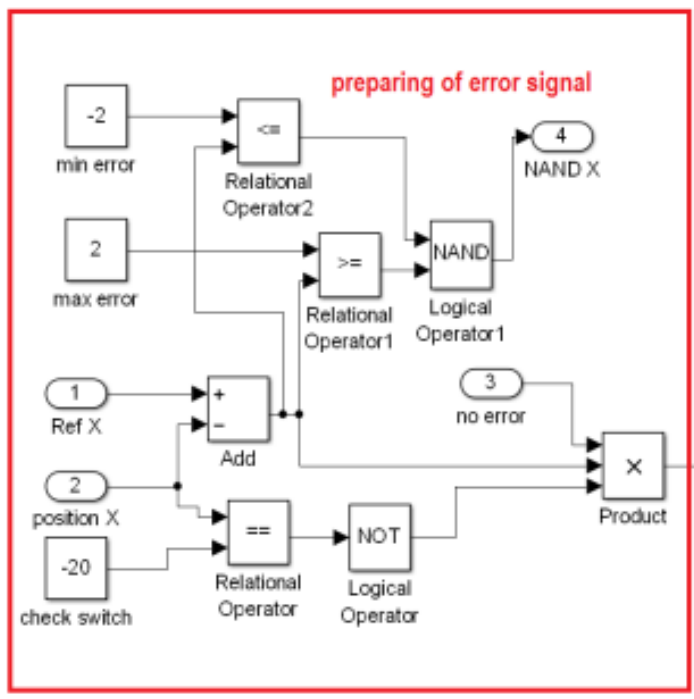

Fig. 9. The control scheme of the control of ball position's in $X$ direction

The parameters of PID controllers are chosen directly on the lab stand in few stages. In first order the system is prepared to tests by setting up the rudder bar of actuators in parallel orientation versus the fixed base of the platform. In result the initial condition of the control system and initial value of control signals are set up to 0 and 90 , respectively. In the next step the ball is located in position $(-55 ;-30)$ from middle position of the touch panel. After that the parameters of the both controllers PIDx and PIDy are tuned by the trial error method. As a result the transfer function of these controllers are expressed as follows:

$P I D_{X}(s)=\frac{0.1 s^{2}+0.35 s+0.25}{s}$

$P I D_{Y}(s)=\frac{0.12 s^{2}+0.45 s+0.25}{s}$

Next, obtained signals from controllers are passed to microcontroller Arduino. The results of carried out investigations - the signals from both control subsystem PID $x$ and PID $y$ (error signal and control signal) were recorded and are shown in Fig. 10 and Fig. 11, respectively.

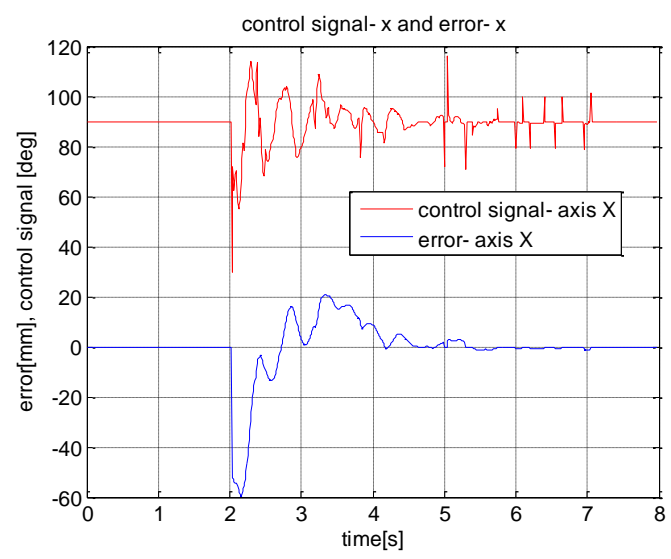

Fig. 10. The control signal and error signal derived by the PID $x$ controller

We can see, that the position of the ball is constant in desired point of the platform up to 2 second. After that, the both control loops are switched on as a result of change of the ball location greater than $2 \mathrm{~mm}$ in two directions. One more time stabilization of the ball of the platform is achieved after 3 or 5 seconds. 
The difference between both transient periods of stabilization of the ball occurs because the controller PIDy generates peaks of high values after 4 seconds in the control signal. Finally, obtained results are satisfying in point of view of control system such structure and further investigations allow us to compare PID controller with other types of control methods.

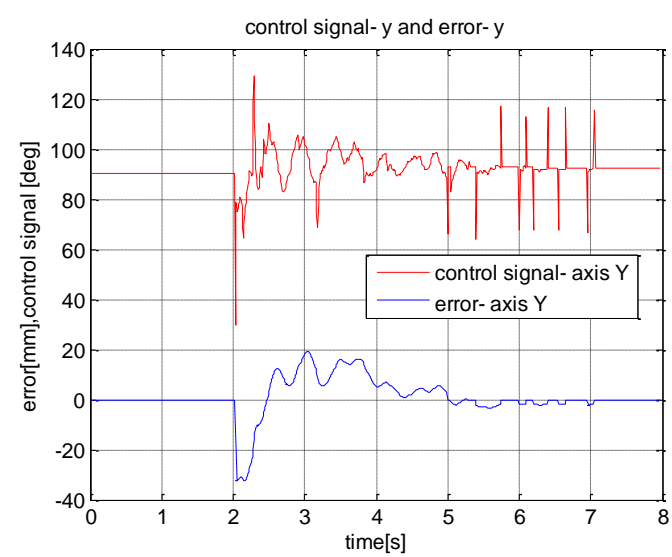

Fig. 11. The control signal and error signal derived by the PIDy controller

\section{CONCLUSIONS}

The research work aimed stabilization of the ball on the Stewart platform with using Arduino microcontroller and Matlab/Simulink software. The process of designation of the parameters of the PID controllers is carried out in many stages. In the first step we successfully shown two process of preparing of the measurment signals: calibration of the touch sensor and filtering signals with using low pass Butterworth filter. Next, the parameters of both PID controllers (PIDx and PIDy) are determined directly on the laboratory stand. Moreover, we passed these parameters of the controllers to microcontroler Arduino and we tested the whole control system in regime of soft-real time system.

\section{REFERENCES}

1. Gough V. E., Whitehall S. G. (2012), Universal tyre test machine, Proceedings of the 9th International Technical Congress F.I.S.I.T.A, 117-137,1962.

2. Paul A. K., Mishra J. K., Radke M. G. (1994, Reduced order sliding mode control for pneumatic actuator, IEEE Transactions on Control Systems Technology, Vol. 2, No. 3, 271-276.

3. Shunmugham P., Hayakawa Y. (1997), Practical design of adaptive model-based sliding mode control of pneumatic actuators, Proceedings of Advanced Intelligent Mechatronics, IEEE, Tokyo, Japan, 140.

4. Urniezius R., Geguzis E. (2014), Hybrid Fuzzy Logic and Adpative LQR Controller for Swing-up, Positioting and Stabilization of Inverted Pendulum, Elektronika ir Elektrotechnika, Vol.20, No.3, 11-15.

5. Varseveld R. B. V., Bone G. M. (1997), Accurate position control of a pneumatic actuator using on/off solenoid valves, IEEE/ASME Transactions on Mechatronics, Vol. 2, No. 3, 195-204.

6. Wang J., Pu J., Moore P. (1999), A practical control strategyfor servo-pneumatic actuator systems, Control Engineering Practice, Vol. 7, No. 12, 1483-1488.

7. Wang W., Yang H., Zou J., Ruan X., Fu X. (2009) Optimal design of Stewart platforms based on expanding the control bandwidth while considering the hydraulic system design, Journal of Zhejiang University, Vol.10(1), 22-30.

8. Weng Ch., Xu Z. (2013), Track-position and vibration control simulation for strut of the Stewart platform, Applied Physics and Engineering, Vol.14(4), 281-291.

9. http://stackoverflow.com/questions/17308956/differences-betweenhard-real-time-soft-real-time-and-firm-real-time (10.09.2014)

10. http://www.buddyrc.com/new-power-xl-9hm.html (12.03.2014)

11. http://www.ece.uah.edu/corses/ee426/Butterworth.pdf (30.09.2014)

This work is co-financed from Dean's Project W/WM/19/2013 and Statutory Work of Department of Automatic Control and Robotics S/WM/1/2012 